\title{
SEN'S THEOREM ON ITERATION OF POWER SERIES
}

\author{
JONATHAN LUBIN
}

(Communicated by Lance W. Small)

\begin{abstract}
In the group of continuous automorphisms of the field of Laurent series in one variable over a field of characteristic $p>0$, Sen's Theorem describes the rapidity of convergence to the identity of the sequence formed by taking successive $p$ th powers of a given element. This paper gives a short proof of Sen's Theorem, utilizing the methods of $p$-adic analysis in characteristic zero.
\end{abstract}

The theorem in question appears in Sen's thesis [Sen], and is concerned with the group $\mathscr{G}_{0,1}(\kappa)$ of formal power series in one variable with no constant term, and first degree coefficient equal to 1 , over a field $\kappa$ of characteristic $p>0$, where the group law is composition of series. If we call the variable $t$, this group is a closed subset of the discrete valuation ring $\kappa[[t]]$, namely, the set of all $u(t)$ for which $u \equiv t\left(\bmod t^{2}\right)$. For the $(t)$-adic filtration of group $\mathscr{G}_{0,1}$, the successive quotients are isomorphic to the additive group $\kappa$. Thus if we call $u^{\circ n}$ the $n$-fold iteration of $u$ with itself, any time that $u \equiv t\left(\bmod t^{n}\right)$, we necessarily have $u^{o p} \equiv t\left(\bmod t^{n+1}\right)$. Sen's Theorem says much more and is best stated in terms of the additive valuation $v$ of $\kappa[[t]]$ normalized so that $v(t)=1$. According to the theorem, if $u^{o p^{n}}$ is not the identity, then $v\left(u^{\circ p^{n}}(t)-t\right) \equiv v\left(u^{\circ p^{n-1}}(t)-t\right)\left(\bmod p^{n}\right)$. Let us abbreviate notation by setting $i_{u}(n)=i(n):=v\left(u^{\circ p^{n}}(t)-t\right)$. Sen's Theorem now says that if $u^{\circ p^{n}}$ is not the identity, then $i(n) \equiv i(n-1)\left(\bmod p^{n}\right)$.

As examples of this phenomenon, we have, in characteristic 2 , if $u(t)=$ $t+t^{4}$, then $i_{u}(n)=2^{2^{n+1}}$; if $u(t)=t+t^{4}+t^{5}$, then $i_{u}(n)=2^{n+2}$; and if $u(t)=$ $t+t^{3}$, then $i_{u}(n)=1+2^{n+1}$. It is easy to see why the first two of these facts hold, since each of $t+t^{4}$ and $t+t^{4}+t^{5}$ is an endomorphism of a formal group, and since in a formal-group endomorphism ring, the multiplication comes from substitution of power series. The first-mentioned series is an endomorphism of the additive formal group $\mathscr{A}(x, y)=x+y$, whose endomorphism ring has characteristic 2 , and in that ring $t+t^{4}$ is $g=1+\phi, \phi(t)=t^{4}$. The powers $g^{2^{i}}$ are

$$
\left(1+\phi^{2^{i}}\right)(t)=t+t^{4^{2^{i}}} .
$$

Received by the editors September 29, 1992 and, in revised form, April 19, 1993.

1991 Mathematics Subject Classification. Primary 12J25; Secondary 11R37, 54H20. 
The second-mentioned series is the endomorphism [5] $]_{\mathscr{M}}(t)$ of the multiplicative formal group $\mathscr{M}(x, y)=x+y+x y$, whose endomorphism ring is isomorphic to the ring $\mathbb{Z}_{2}$ of 2 -adic integers, and the iterates of [5] $(t)$ approach $[1](t)=t$ in the manner claimed because of the congruences $5^{2^{n}} \equiv 1\left(\bmod 2^{n+2}\right), 5^{2^{n}} \not \equiv 1$ $\left(\bmod 2^{n+3}\right)$. To see why the 2-power iterates of the last-mentioned series $t+t^{3}$ approach the identity in the manner claimed is rather more difficult, and for this the reader is referred to $[\mathrm{K}]$.

In this note we give a short proof of Sen's Theorem using the methods of $p$-adic analysis.

Without loss of generality, we may assume that the field $\kappa$ is perfect. The trick is to lift $u$ in a particular way to a series $U(x)$ in characteristic zero. (The choice of a complete discrete valuation ring $\mathfrak{o}$ of characteristic zero to serve as constant ring for $U$ is not crucial: the Witt ring $W_{\infty}(\kappa)$ will do.) As usual in $p$-adic analysis, we pass from the original ground ring $o$ to its integral closure $\mathfrak{D}$ in an algebraic closure of the fraction field $k$ of $\mathfrak{o}$. Of course, $\mathfrak{D}$ is neither Noetherian nor complete, but every series considered will have its coefficients in a finite algebraic extension of $k$, in which the integer ring is complete and Noetherian. Call $\mathfrak{M}$ the maximal ideal of $\mathfrak{D}$. The number $i(n)$ defined above is now the "Weierstrass degree" of the series $U^{o p^{n}}(t)-t$, and $i(n)$ is thus the number of fixed points in $\mathfrak{M}$ of $U^{o p^{n}}$, taking account of multiplicity. The idea is to choose the series $U$ so that each periodic point of order dividing $p^{n}$ has multiplicity at most one in every iterate of $U$. The existence of such a series will make a proof of Sen's Theorem easy. The note closes with a construction of the series $U$.

Theorem. Let $\mathfrak{o}$ be a complete discrete valuation ring of characteristic zero, maximal ideal $\mathfrak{m}$, and residue field $\kappa$ of characteristic $p>0$. Let $U(t)$ be a series in $0[[t]]$ for which $U(0)=0$, and suppose that $n$ is a positive integer such that $U^{\circ p^{n}}(t) \not \equiv t(\bmod \mathfrak{m})$ and all roots of $U^{\circ p^{n}}(t)-t$ in $\mathfrak{M}$ are simple. Then for all $m$ with $0<m \leq n, i_{U}(m-1) \equiv i_{U}(m)\left(\bmod p^{m}\right)$.

Proof. For each $m \geq 1$ let $Q_{m}(t)$ be defined by

$$
Q_{m}(t)=\frac{U^{\circ p^{m}}(t)-t}{U^{\circ p^{m-1}}(t)-t} .
$$

The quotient is a series in $\mathfrak{o}[[t]]$ since for any series $f \in \mathfrak{o}[[t]]$ with $f(0)=0$ we have $(f(t)-t) \mid\left(f^{\circ r}(t)-t\right)$. Put $Q_{0}(t)=U(t)-t$. Our hypothesis on multiplicities says that no two of the series $Q_{0}, Q_{1}, \ldots, Q_{n}$ have any roots in common. Thus the set of roots of $Q_{m}$ in $\mathfrak{M}$ is exactly the set of points of $\mathfrak{M}$ that lie in an orbit of cardinality $p^{m}$ under the action of $U$. Since, for $m \geq 1$, the Weierstrass degree of $Q_{m}$ is $i_{U}(m)-i_{U}(m-1)$, the proof is done.

All the difficulty in Sen's Theorem is pushed into the construction of a lifting of the given $u(t) \in \kappa[[t]]$ to a series $U(t) \in \mathfrak{o}[[t]]$ of the desired form.

Proposition. Let $\kappa$ be a field of characteristic $p>0$, and let $u$ be a series in $\kappa[[t]]$ with $u(t) \equiv t\left(\bmod t^{2}\right)$. If $n$ is an integer such that $u^{o p^{n}}(t) \neq t$, then there is a complete discrete valuation ring $(\mathfrak{o}, \mathfrak{m})$ of characteristic zero, such that $\mathfrak{o} / \mathfrak{m}$ contains $\kappa$, and a lifting $U$ of $u$ to $o[[t]]$, such that all the roots of $U^{\circ p^{n}}(t)-t$ in $\mathfrak{M}$ are simple. 
Proof. First we find any complete discrete valuation ring at all, $\left(\mathfrak{o}_{0}, \mathfrak{m}_{0}\right)$, whose residue field contains $\kappa$ : the Witt ring of the perfect closure of $\kappa$ will do. Lift $u$ in any way to a series $U_{0} \in o_{0}[[t]]$ without constant term. Our strategy is to choose a ring $(\mathfrak{o}, \mathfrak{m})$ that is the integer ring of a finite algebraic extension of the fraction field of $\mathfrak{o}_{0}$ and modify $U_{0}$ by adding a carefully chosen $\Delta \in p^{N} \mathrm{o}[[t]]$ so that $U=U_{0}+\Delta$ satisfies the desired conditions. We make frequent use of the continuity of the roots of a series over $\mathfrak{o}$, by which we mean that if $\xi f(t) \in \mathfrak{o}[[\xi]][[t]]$ and if $\rho \in \mathfrak{m}$ is a root of multiplicity $\mu$ of $0 f$, then for all $\alpha$ in a sufficiently high power of $\mathfrak{m}$, there are precisely $\mu$ roots of ${ }_{\alpha} f$, counting multiplicity, that correspond to $\rho$. In particular, when $f$ is varied slightly in a suitably small open set about of the multiplicities of roots cannot increase.

We recall also that a fixed point $\zeta$ of $f(t)$ has multiplicity greater than 1 if and only if $f^{\prime}(\zeta)=1$ and that $\zeta$ will be a multiple root of $f^{\circ}(t)-t$ if and only if $f^{\prime}(\zeta)$ is an $r$ th root of 1 . The last tool used in the proof is the observation that if $\Delta \in \mathfrak{o}[[t]]$ is a series that vanishes at all roots of $U_{0}^{o p^{n}}(x)-x$ and if $U=U_{0}+\Delta$, then every fixed point of $U_{0}^{o p^{n}}$ is a fixed point of $U^{\circ p^{n}}$. We will modify the original $U_{0}$ in this way by increments that successively decrease the multiplicity of each fixed point of $U^{o p^{n}}$ to 1 . Note that our modified series has only finitely many periodic points of order dividing $p^{n}$ since $u^{\circ p^{n}}(t) \neq t$.

Now for the details: In case a fixed point $\zeta$ of $U$ itself is a fixed point of multiplicity greater than 1 in an iterate, we may assume (after perhaps making a finite extension of the base) that $\zeta=0$, so that $U^{\circ p^{n}}(t)-t$ takes the form $t^{e} G(t)$, with $G(0) \neq 0$ and $e>1$. The hypothesis on $U$ is that $U^{\prime}(0)=w$ is a root of 1 , so we set ${ }_{\xi} U(t):=U(t)+\xi t G(t)$, which, for small enough nonzero $\xi$, has ${ }_{\xi} U^{\prime}(0) \neq w$, but so close to $w$ that it cannot be a root of 1 . Therefore, no iterate of the new series has a fixed point of multiplicity greater than 1 at 0 .

A slightly more complicated situation is the one where $\zeta$ is a periodic point of order $p^{r}$, with $1 \leq r \leq n$. Call $\zeta_{i}:=U^{\circ i}(\zeta)$, so that $\zeta_{i} \neq \zeta$ if $0<i<p^{r}$. The hypothesis on $\zeta$ implies that

$$
U^{\circ p^{n}}(t)-t=G(t) \prod_{i=0}^{p^{r}-1}\left(t-\zeta_{i}\right)^{e_{i}}
$$

where $G$ is nonzero at all the $\zeta$ 's and where $e_{0}>1$. We now set $\Delta(t)$ equal to the series $G(t)(t-\zeta) \prod_{i \neq c}\left(t-\zeta_{i}\right)^{2}$ and set ${ }_{\xi} U:=U+\xi \Delta$. This has among its periodic points of order dividing $p^{n}$ the corresponding periodic points of $U$, and since the hypothesis on $\zeta$ implies that $U^{\circ p^{\prime \prime}}(\zeta)=w$, a root of 1 , we will be done when we show that we can adjust $\xi$ so that ${ }_{\xi} U^{\circ p^{\prime \prime}}(\zeta)$ is so close to $w$ that it cannot be a root of 1 . We have

$$
\begin{aligned}
{ }_{\xi} U^{\circ p^{\prime \prime}}(\zeta) & =\prod_{i=0}^{p^{r}-1} U^{\prime}\left(\xi U^{\circ i}(\zeta)\right)={ }_{\xi} U^{\prime}(\zeta) \prod_{i=1}^{p^{r}-1} U_{\xi}\left(\zeta_{i}\right) \\
& =\left(U^{\prime}(\zeta)+\xi \Delta^{\prime}(\zeta)\right) \prod_{i=1}^{p^{r}-1} U^{\prime}\left(\zeta_{i}\right)=w+\xi \Delta^{\prime}(\zeta) \prod_{i=1}^{p^{r}-1} U^{\prime}\left(\zeta_{i}\right),
\end{aligned}
$$

and since we have constructed $\Delta$ so that $\Delta^{\prime}(\zeta) \neq 0$, the proof is done. 


\section{REFERENCES}

[K] K. Keating, Automorphisms and extensions of $k((t))$, J. Number Theory 41 (1992), 314321.

[Sen] S. Sen, On automorphisms of local fields, Ann. of Math. (2) 90 (1969), 33-46.

Department of Mathematics, Brown University, Box 1917, Providence, Rhode Island 02912

E-mail address: ma406000@brownvm.brown.edu 